\title{
Meningitis: Causes, Diagnosis, symptoms, Treatment and prevention
}

\author{
S vanisree reddy* \\ B.pharmacy, Balaji College of pharmacy, Anantapur, Andra pradesh, India
}

Received: December 02, 2016; Accepted: December 06, 2016; Published: December 12, 2016

*Corresponding author: S.vanisree reddy, B.pharmacy, Balaji College of pharmacy, Anantapur, Andra pradesh, India, Tel:9000937470, E-mail: vanisreereddy44@gmail.com

\begin{abstract}
Bacterial meningitis continues to be a significant source of morbidity and mortality worldwide. Streptococcus pneumoniae and Neisseria meningitidis are the most ordinary and most violent pathogens of meningitis. Clinical and experimental studies have recognized a more detailed understanding of the mechanisms resulting in brain injure, squeal and neuropsychological deficit. Vaccination has really contributed to the current turn down in the number of cases for the three most usual meningeal pathogens. Genetic targeting and/or pharmacologic blockade of the implicated pathways possibly a potential strategy for therapeutic adjunctive measures to improve outcome and may hold substantial assure, in concert with antimicrobial agents, in human with delicate bacterial meningitis
\end{abstract}

Keywords: Streptococcus pneumonia, Neisseria meningitides, Haemophilus influenzae

\section{Transmission}

Meningococcal ailment is spread from one person to another person through droplets of respiratory or gullet secretions from carriers or lengthy contact, fortunately, these bacteria are not as contagious as germs that cause the regular cold or the influenza. The average incubation period is four days, but can the range between two to ten days.

Neisseria meningitidis only infects humans not spared to animals. People in the living same house or roommates or lip kisses, coughing would be considered at increased risk of getting the infection.

\section{Diagnosis}

Early analysis and treatment are exceedingly significant, and an elevated index of clinical suspicion is necessary.

\section{Symptoms}

- $\quad$ Nausea

- $\quad$ Vomiting

- Cold

\section{- $\quad$ Cough}

- Headache and fever

- Viewing a pale, dappled complexion

- $\quad$ Rash (red or purple pinpricks)

\section{Treatment}

Immediate antibiotic therapy is imperative and must not be postponed by diagnostic delays and prior to dealing, a blood civilization should be obtained. Since microbiological identification to the pathogen is not immediately obtainable, the first choice of antibiotics is regularly empirical. Factors to consider include regional antibiotic resistance rates, patient period, predisposing conditions and resources

Corticosteroids decrease brain edema, intracranial hypertension and meningeal inflammation in experimental models of bacterial meningitis. In adults, a single double-blind RCT of 301 adult patients reported compact mortality and lower frequency of hear trouncing and neuropsychological sequelae. The community-acquired meningitis of children $(0.15 \mathrm{mg} / \mathrm{kg}$ every six hours for two to four days) and adults (10 mg each 5 or 6 hours for 4 to 5 days) Discontinuation of this therapy is advisable Children are routinely immunized with the Hemophilus influenzae type b (Hib) vaccine and Neisseria meningitides, Streptococcus pneumonia (adults and children) can be ruled out as the underlying pathogen.

\section{Prevention}

- Don't share drinks or any other fluids, food, eating utensils, lip balms or toothbrushes with anyone else.

- Bed Rest

- Cold Packs

- Neutral Bath

- Drinking hot water

- Avoid relationships

- Cover your mouth

- Careful your hand wash its prevent germs 


\section{Discussion}

I was told that meningitis is a highly infected disease it bags of meningitis are caused by microorganisms, such as virus, fungus, germs or bedbugs that increase into the blood and into the cerebrospinal fluid (CSF) [1]. Non-infectious causes include cancers, convinced drugs and more.

\section{Conclusion}

Bacterial meningitis reaches all community stratum and the deprived living conditions have a better proportion of cases that progress to loss. This judgment reflects the difficulties for prepared access and low quality of medical care faced by these populations.

\section{References}

1. Catherine L. Tacon, Oliver Flower. Diagnosis and Management of Bacterial Meningitis in the Paediatric Population: A Review. Emergency Medicine International. 2012 (2012), 320309:8.

2. Olaf Hoffman, R. Joerg Weber. Pathophysiology and Treatment of Bacterial Meningitis. Ther Adv Neurol Disord. 2009;2(6): 1-7.

3. Souza SF, Costa Mda C, Paim JS, Natividade MS, Pereira SM, Andrade $\mathrm{AM}$, et al. Bacterial meningitis and living conditions. Rev Soc Bras Med Trop. 2012;45(3):323-8. 